\title{
On conjugacy of maximal abelian subalgebras and the outer automorphism group of the Cuntz algebra
}

\author{
Roberto Conti \\ Dipartimento di Scienze di Base e Applicate per l'Ingegneria, \\ 00161 Roma, Italy (roberto.conti@sbai.uniroma1.it) \\ Jeong Hee Hong \\ Department of Data Information, \\ Korea Maritime and Ocean University, Busan 606-791, \\ Republic of Korea (hongjh@kmou.ac.kr)

\section{Wojciech Szymański} \\ Department of Mathematics and Computer Science, \\ University of Southern Denmark, Campusvej 55, \\ 5230 Odense M, Denmark (szymanski@imada.sdu.dk)
} Sezione di Matematica, Sapienza Università di Roma, Via A. Scarpa 16,

(MS received 27 August 2013; accepted 17 December 2013)

We investigate the structure of the outer automorphism group of the Cuntz algebra and the closely related problem of conjugacy of maximal abelian subalgebras in $\mathcal{O}_{n}$. In particular, we exhibit an uncountable family of maximal abelian subalgebras, conjugate to the standard maximal abelian subalgebra $\mathcal{D}_{n}$ via Bogolubov automorphisms, that are not inner conjugate to $\mathcal{D}_{n}$.

\section{Introduction}

The main motivation for the present paper comes from the desire to better understand the structure of the outer automorphism group of the Cuntz algebra $\mathcal{O}_{n}[12$, 14]. As the Cuntz algebras are among the most intensely investigated operator algebras, it is not surprising that both their single automorphisms and the structure of their automorphism groups have attracted a lot of interest. In addition to the obvious intrinsic value of this line of research, we would also like to point out its importance for the current efforts within Elliott's classification programme. In this context, for example, the question as to whether $\operatorname{Aut}\left(\mathcal{O}_{2}\right)$ is a universal Polish group is raised in [25, question 9.1].

Our point of departure for the investigations of $\operatorname{Out}\left(\mathcal{O}_{n}\right)$ is the recent progress in the understanding of $\operatorname{Aut}\left(\mathcal{O}_{n}, \mathcal{D}_{n}\right)$, the group of those automorphisms of $\mathcal{O}_{n}$ that globally preserve the standard maximal abelian subalgebra (MASA) $\mathcal{D}_{n}[8,11]$. This group has the structure of a semi-direct product $\lambda\left(\mathcal{U}\left(\mathcal{D}_{n}\right)\right) \rtimes \lambda\left(\mathcal{S}_{n}\right)^{-1}$, where $\lambda\left(\mathcal{U}\left(\mathcal{D}_{n}\right)\right)$ is a maximal abelian subgroup of $\operatorname{Aut}\left(\mathcal{O}_{n}\right)$ of those automorphisms that

(C) 2015 The Royal Society of Edinburgh 
fix $\mathcal{D}_{n}$ pointwise (see [13, proposition 1.3]) and $\lambda\left(\mathcal{S}_{n}\right)^{-1}$ is countable and discrete, the so-called Weyl group of $\mathcal{O}_{n}$. Of particular note here is the relation between the image of $\lambda\left(\mathcal{S}_{n}\right)^{-1}$ in $\operatorname{Out}\left(\mathcal{O}_{n}\right)$ and the group of automorphisms of the full two-sided $n$-shift shown in [10].

The next logical step in the study of $\operatorname{Out}\left(\mathcal{O}_{n}\right)$ would be to learn if every automorphism of $\mathcal{O}_{n}$ has a representative in $\operatorname{Out}\left(\mathcal{O}_{n}\right)$ coming from $\operatorname{Aut}\left(\mathcal{O}_{n}, \mathcal{D}_{n}\right)$, and, if not, to classify MASAs of $\mathcal{O}_{n}$ that are outer, but not inner, conjugate to $\mathcal{D}_{n}$ (that is, one is mapped onto the other by an outer automorphism but no such inner automorphism exists). In fact, this question was raised a few years ago by Joachim Cuntz in a conversation with the third author. In the present paper, we show that Bogolubov automorphisms either globally preserve $\mathcal{D}_{n}$ or move it to other, not inner conjugate, MASAs; see theorem 3.7 and corollary 3.8.

Naturally, any investigations of the structure of an outer automorphism group are significantly helped by classification of single automorphisms up to conjugacy. In the context of the Cuntz algebras, a great deal of progress has been achieved in this direction and we would like to specifically call the attention of the reader to $[18,19$, 21]. By [21, theorem 9] and [24, theorem 3.6], any two aperiodic automorphisms of $\mathcal{O}_{n}$ are outer conjugate, which is in nice analogy with the classification of aperiodic automorphisms of the hyperfinite $I I_{1}$ factor due to Connes [4]. The classification of non-aperiodic automorphisms of $\mathcal{O}_{n}$ is also related to the seminal work of Connes [5], although in this case the $C^{*}$-algebraic setting is much more intricate by comparison with the von Neumann algebraic one. Indeed, non-aperiodic automorphisms of the hyperfinite $I I_{1}$ factor are completely classified by pairs $(k, \gamma)$, with $k$ a positive integer (outer period) and $\gamma$ a $k$ th root of unity [5, theorem 1.5]. It is shown in [5, proposition 1.6] that each invariant $(k, \gamma)$ may be realized in $\operatorname{Aut}\left(\mathcal{O}_{k}\right)$ by an automorphim of the form $\lambda_{d} \lambda_{w}$ with $d$ a unitary in the canonical MASA $\mathcal{D}_{n}$ and $\lambda_{w}$ a Bogolubov permutation. However, there exist automorphisms of $\mathcal{O}_{2}$ with $k=2$ and $\gamma=1$ that are not outer conjugate to each other [18, example 5.8]. In the present paper, classification results for single automorphisms of $\mathcal{O}_{n}$ are used in the proofs of several structural properties of $\operatorname{Out}\left(\mathcal{O}_{n}\right)$ collected in $\S 4$.

\section{Notation and preliminaries}

If $n$ is an integer greater than 1 , then the Cuntz algebra $\mathcal{O}_{n}$ is a unital simple purely infinite $C^{*}$-algebra generated by $n$ isometries $S_{1}, \ldots, S_{n}$ satisfying $\sum_{i=1}^{n} S_{i} S_{i}^{*}=$ 1 [12]. We denote by $W_{n}^{k}$ the set of $k$-tuples $\mu=\left(\mu_{1}, \ldots, \mu_{k}\right)$ with $\mu_{m} \in\{1, \ldots, n\}$, and by $W_{n}$ the union $\bigcup_{k=0}^{\infty} W_{n}^{k}$, where $W_{n}^{0}=\{0\}$. If $\mu \in W_{n}^{k}$, then $|\mu|=k$ is the length of $\mu$. If $\mu=\left(\mu_{1}, \ldots, \mu_{k}\right) \in W_{n}$ then $S_{\mu}=S_{\mu_{1}} \ldots S_{\mu_{k}}\left(S_{0}=1\right.$ by convention) is an isometry with range projection $P_{\mu}=S_{\mu} S_{\mu}^{*}$. Every word in $\left\{S_{i}, S_{i}^{*} \mid i=1, \ldots, n\right\}$ can be uniquely expressed as $S_{\mu} S_{\nu}^{*}$ for $\mu, \nu \in W_{n}[12$, lemma 1.3].

We denote by $\mathcal{F}_{n}^{k}$ the $C^{*}$-subalgebra of $\mathcal{O}_{n}$ spanned by all words of the form $S_{\mu} S_{\nu}^{*}, \mu, \nu \in W_{n}^{k}$, which is isomorphic to the matrix algebra $M_{n^{k}}(\mathbb{C})$. The norm closure $\mathcal{F}_{n}$ of $\bigcup_{k=0}^{\infty} \mathcal{F}_{n}^{k}$ is the uniformly hyperfinite algebra (UHF-algebra) of type $n^{\infty}$ [16], called the core UHF-subalgebra of $\mathcal{O}_{n}$ [12]. We denote by $\tau$ the unique normalized trace on $\mathcal{F}_{n}$. Subalgebra $\mathcal{F}_{n}$ is the fixed-point algebra for the gauge action $\gamma: U(1) \rightarrow \operatorname{Aut}\left(\mathcal{O}_{n}\right)$, such that $\gamma_{z}\left(S_{j}\right)=z S_{j}$ for $z \in U(1)$ and $j=1, \ldots, n$. 
For an integer $m \in \mathbb{Z}$, we define

$$
\mathcal{O}_{n}^{(m)}:=\left\{x \in \mathcal{O}_{n}: \gamma_{z}(x)=z^{m} x \forall z \in U(1)\right\},
$$

a spectral subspace for $\gamma$. Then $\mathcal{O}_{n}^{(0)}=\mathcal{F}_{n}$ and for each positive integer $m$ and each $\alpha \in W_{n}^{m}$ we have $\mathcal{O}_{n}^{(m)}=\mathcal{F}_{n} S_{\alpha}$ and $\mathcal{O}_{n}^{(-m)}=S_{\alpha}^{*} \mathcal{F}_{n}$. Furthermore,

$$
E_{m}(x)=\int_{z \in U(1)} z^{-m} \gamma_{z}(x) \mathrm{d} z
$$

is a completely contractive projection from $\mathcal{O}_{n}$ onto $\mathcal{O}_{n}^{(m)}$, such that $E_{m}($ xay $)=$ $x E_{m}(a) y$ for all $a \in \mathcal{O}_{n}, x, y \in \mathcal{F}_{n}$. In particular, $E:=E_{0}$ is the faithful conditional expectation from $\mathcal{O}_{n}$ onto $\mathcal{F}_{n}$ given by averaging action $\gamma$ over $U(1)$ with respect to the Haar measure.

The $C^{*}$-subalgebra of $\mathcal{O}_{n}$ generated by projections $P_{\mu}, \mu \in W_{n}$, is a MASA in $\mathcal{O}_{n}$. We call it the diagonal and denote it by $\mathcal{D}_{n}$, also writing $\mathcal{D}_{n}^{k}$ for $\mathcal{D}_{n} \cap \mathcal{F}_{n}^{k}$. The spectrum of $\mathcal{D}_{n}$ is naturally identified with $X_{n}$, the full one-sided $n$-shift space (a Cantor set). Occasionally, we will view $X_{n}$ as a metric space equipped with the metric $\operatorname{dist}(x, y)=n^{-k}$, where $k=\min \left\{m \in \mathbb{N} \mid x_{m} \neq y_{m}\right\}$.

As shown by Cuntz in [13], there exists the following bijective correspondence between unitaries in $\mathcal{O}_{n}$ (whose collection is denoted by $\mathcal{U}\left(\mathcal{O}_{n}\right)$ ) and unital *-endomorphisms of $\mathcal{O}_{n}$ (whose collection we denote by $\operatorname{End}\left(\mathcal{O}_{n}\right)$ ), determined by

$$
\lambda_{u}\left(S_{i}\right)=u S_{i}, \quad i=1, \ldots, n .
$$

Composition of endomorphisms corresponds to the 'convolution' multiplication of unitaries: $\lambda_{u} \circ \lambda_{w}=\lambda_{\lambda_{u}(w) u}$. In the case $u, w \in \mathcal{U}\left(\mathcal{F}_{n}^{1}\right)$, this formula simplifies to $\lambda_{u} \circ \lambda_{w}=\lambda_{u w}$ and, in particular, there exists an imbedding $u \mapsto \lambda_{u}$ of $U(n) \cong \mathcal{U}\left(\mathcal{F}_{n}^{1}\right)$ into $\operatorname{Aut}\left(\mathcal{O}_{n}\right)$ [15]. If $A$ is either a unital $C^{*}$-subalgebra of $\mathcal{O}_{n}$ or a subset of $\mathcal{U}\left(\mathcal{O}_{n}\right)$, then we denote $\lambda(A)=\left\{\lambda_{u} \in \operatorname{End}\left(\mathcal{O}_{n}\right): u\right.$ unitary in $\left.A\right\}$ and $\lambda(A)^{-1}=\left\{\lambda_{u} \in \operatorname{Aut}\left(\mathcal{O}_{n}\right): u\right.$ unitary in $\left.A\right\}$.

We denote by $\varphi$ the canonical shift on the Cuntz algebra

$$
\varphi(x)=\sum_{i=1}^{n} S_{i} x S_{i}^{*}, \quad x \in \mathcal{O}_{n} .
$$

Clearly, $\varphi\left(\mathcal{F}_{n}\right) \subset \mathcal{F}_{n}$ and $\varphi\left(\mathcal{D}_{n}\right) \subset \mathcal{D}_{n}$. We denote by $\sigma: X_{n} \rightarrow X_{n}$ the shift on $X_{n}$. Then we have $\varphi(f)(x)=f(\sigma(x))$ for all $f \in C\left(X_{n}\right)$ and $x \in X_{n}$.

For all $u \in \mathcal{U}\left(\mathcal{O}_{n}\right)$, we have $\operatorname{Ad}(u)=\lambda_{u \varphi\left(u^{*}\right)}$. If $u \in \mathcal{U}\left(\mathcal{O}_{n}\right)$, then for each positive integer $k$ we define

$$
u_{k}=u \varphi(u) \cdots \varphi^{k-1}(u) \text {. }
$$

Here, $\varphi^{0}=\mathrm{id}$, and we agree that $u_{k}^{*}$ stands for $\left(u_{k}\right)^{*}$. If $\alpha$ and $\beta$ are multi-indices of length $k$ and $m$, respectively, then $\lambda_{u}\left(S_{\alpha} S_{\beta}^{*}\right)=u_{k} S_{\alpha} S_{\beta}^{*} u_{m}^{*}$. This is established through a repeated application of the identity $S_{i} x=\varphi(x) S_{i}$, valid for all $i=1, \ldots, n$ and $x \in \mathcal{O}_{n}$.

We often consider elements of $\mathcal{O}_{n}$ of the form $w=\sum_{(\alpha, \beta) \in \mathcal{J}} c_{\alpha, \beta} S_{\alpha} S_{\beta}^{*}$, where $\mathcal{J}$ is a finite collection of pairs $(\alpha, \beta)$ of words $\alpha, \beta \in W_{n}$ and $c_{\alpha, \beta} \in \mathbb{C}$. In particular, we consider the group $\mathcal{S}_{n}$ of those unitaries in $\mathcal{O}_{n}$ that can be written as finite sums of words, i.e. in the form $w=\sum_{(\alpha, \beta) \in \mathcal{J}} S_{\alpha} S_{\beta}^{*}$. Each $w \in \mathcal{S}_{n}$ normalizes $\mathcal{D}_{n}$, 
and hence $\lambda_{w}\left(\mathcal{D}_{n}\right) \subseteq \mathcal{D}_{n}\left[13\right.$, proposition 1.4]. We define $\mathcal{P}_{n}:=\mathcal{S}_{n} \cap \mathcal{U}\left(\mathcal{F}_{n}\right)$ and $\mathcal{P}_{n}^{k}:=\mathcal{S}_{n} \cap \mathcal{U}\left(\mathcal{F}_{n}^{k}\right)$. It is known that $\mathcal{S}_{2}$ is isomorphic to the Thompson group $V$ [22, proposition 9.6].

For algebras $A \subseteq B$ we denote by $\mathcal{N}_{B}(A)=\left\{u \in \mathcal{U}(B): u A u^{*}=A\right\}$ the normalizer of $A$ in $B$ and by $A^{\prime} \cap B=\{b \in B:(\forall a \in A) a b=b a\}$ the relative commutant of $A$ in $B$. We also denote by $\operatorname{Aut}(B, A)$ the collection of all those automorphisms $\alpha$ of $B$ such that $\alpha(A)=A$, and by $\operatorname{Aut}_{A}(B)$ those automorphisms of $B$ that fix $A$ pointwise. Likewise, we denote by $\operatorname{End}_{A}(B)$ the collection of those endomorphisms of $B$ that fix pointwise subalgebra $A$.

\section{Conjugacy of MASAs}

If $A_{1}$ and $A_{2}$ are two MASAs in $B$, then we say they are conjugate if there exists an $\alpha \in \operatorname{Aut}(B)$ such that $\alpha\left(A_{1}\right)=A_{2}$. We say $A_{1}$ and $A_{2}$ are inner conjugate if there exists a $u \in \mathcal{U}(B)$ such that $u A_{1} u^{*}=A_{2}$.

Proposition 3.1. Let $z \in \mathcal{U}\left(\mathcal{O}_{n}\right)$ be such that $\lambda_{z} \in \operatorname{Aut}\left(\mathcal{O}_{n}\right)$. Then there exists a $u \in \mathcal{U}\left(\mathcal{O}_{n}\right)$ such that

$$
\lambda_{z}\left(\mathcal{D}_{n}\right)=\operatorname{Ad}(u)\left(\mathcal{D}_{n}\right)
$$

if and only if there exist $u \in \mathcal{U}\left(\mathcal{O}_{n}\right)$ and $w \in \mathcal{S}_{n}$ such that

$$
\lambda_{z}(d)=\operatorname{Ad}(u) \lambda_{w}(d) \quad \forall d \in \mathcal{D}_{n} .
$$

Proof. Suppose that (3.1) holds. Then $\operatorname{Ad}\left(u^{*}\right) \lambda_{z} \in \operatorname{Aut}\left(\mathcal{O}_{n}, \mathcal{D}_{n}\right)$, and thus there exist $v \in \mathcal{U}\left(\mathcal{D}_{n}\right)$ and $w \in \mathcal{S}_{n}$ such that $\operatorname{Ad}\left(u^{*}\right) \lambda_{z}=\lambda_{v} \lambda_{w}$ [8, theorem 2.1]. Since $\left.\lambda_{v}\right|_{\mathcal{D}_{n}}=$ id and $\lambda_{w}\left(\mathcal{D}_{n}\right)=\mathcal{D}_{n}$, for each $d \in \mathcal{D}_{n}$ we have $\operatorname{Ad}\left(u^{*}\right) \lambda_{z}(d)=\lambda_{v} \lambda_{w}(d)=$ $\lambda_{w}(d)$, and identity (3.2) holds.

Conversely, suppose that (3.2) holds. Then $\lambda_{z}^{-1} \operatorname{Ad}(u) \lambda_{w} \in \operatorname{End}_{\mathcal{D}_{n}}\left(\mathcal{O}_{n}\right)$, and thus there exists a $v \in \mathcal{U}\left(\mathcal{D}_{n}\right)$ such that $\lambda_{z}^{-1} \operatorname{Ad}(u) \lambda_{w}=\lambda_{v}$ (see [6, proposition 3.2]). Since $\lambda\left(\mathcal{D}_{n}\right) \subseteq \operatorname{Aut}\left(\mathcal{O}_{n}\right)$ (see [13, proposition 1.3]), $\lambda_{w}$ is an automorphism of $\mathcal{O}_{n}$. Since $\lambda_{w}\left(\mathcal{D}_{n}\right) \subseteq \mathcal{D}_{n}$ and $\mathcal{D}_{n}$ is a MASA in $\mathcal{O}_{n}$, we may conclude that $\lambda_{w}\left(\mathcal{D}_{n}\right)=\mathcal{D}_{n}$, and identity (3.1) follows.

Before proving our main result, theorem 3.7, we need some preparation.

LEMMA 3.2. If $x \in \mathcal{O}_{n}, x \geqslant 0$, and $x \mathcal{D}_{n}=\mathcal{D}_{n} x$, then $x \in \mathcal{D}_{n}$.

Proof. We may assume that $0 \leqslant x \leqslant 1$. Let $\Phi$ be the faithful conditional expectation from $\mathcal{O}_{n}$ onto $\mathcal{D}_{n}$. Since $0 \leqslant x^{2} \leqslant x$, we have $0 \leqslant \Phi\left(x^{2}\right) \leqslant \Phi(x)$. Let $d \in \mathcal{D}_{n}$ be such that $d \Phi(x)=0$. Then $0 \leqslant d \Phi\left(x^{2}\right) d^{*} \leqslant d \Phi(x) d^{*}=0$, and hence $\Phi\left(d x^{2} d^{*}\right)=$ $d \Phi\left(x^{2}\right) d^{*}=0$. Consequently, $d x=0$. Now, for an arbitrary $a \in \mathcal{D}_{n}$, let $b \in \mathcal{D}_{n}$ be such that $x a=b x$. Then $(a-b) \Phi(x)=0$, and thus $(a-b) x=0$. This shows that $x$ is in the commutant of $\mathcal{D}_{n}$, and therefore $x \in \mathcal{D}_{n}$.

REMARK 3.3. The conclusion of lemma 3.2 remains valid if $\mathcal{D}_{n} \subseteq \mathcal{O}_{n}$ are replaced by any $C^{*}$-algebras $D \subseteq A$ such that $D$ is a MASA in $A$ and there exists a faithful conditional expectation from $A$ onto $D$. However, it may fail if $x$ is merely selfadjoint but not positive. For example, simply take $A=M_{2}(\mathbb{C}), D$ the diagonal matrices and $x$ the matrix with both off-diagonal entries equal to 1 and the diagonal ones both 0 . 
Lemma 3.4. Let $z \in \mathcal{U}\left(\mathcal{F}_{n}^{1}\right) \backslash \mathcal{N}_{\mathcal{F}_{n}^{1}}\left(\mathcal{D}_{n}^{1}\right)$ and $x \in \mathcal{F}_{n}$. If $\lambda_{z}\left(\mathcal{D}_{n}\right) x=x \mathcal{D}_{n}$, then $x=0$.

Proof. Since $z \in \mathcal{U}\left(\mathcal{F}_{n}^{1}\right) \backslash \mathcal{N}_{\mathcal{F}_{n}^{1}}\left(\mathcal{D}_{n}^{1}\right)$, there exist a minimal projection $p \in \mathcal{D}_{n}^{1}$ and a $\delta>0$ such that, for all $m \in \mathbb{N}$, all projections $e \in \mathcal{D}_{n}^{m}$ and all $h \in \mathcal{D}_{n}$, we have

$$
\left\|e \lambda_{z}\left(\varphi^{m}(p)\right)-h\right\| \geqslant \delta .
$$

Indeed, since $z \notin \mathcal{N}_{\mathcal{F}_{n}^{1}}\left(\mathcal{D}_{n}^{1}\right)$, there exists an $i \in W_{n}^{1}$ such that $\lambda_{z}\left(P_{i}\right) \notin \mathcal{D}_{n}$. By the Hahn-Banach theorem, there exists a functional $\omega$ of norm 1 on $\mathcal{O}_{n}$ such that $\omega\left(\lambda_{z}\left(P_{i}\right)\right)=\delta>0$ and $\left.\omega\right|_{\mathcal{D}_{n}}=0$. Now, take $m \in \mathbb{N}$, a projection $e \in \mathcal{D}_{n}^{m}$ and an $h \in \mathcal{D}_{n}$, and let $\alpha \in W_{n}^{m}$ be such that $e P_{\alpha}=P_{\alpha}$. Then

$$
\begin{aligned}
\left\|e \lambda_{z}\left(\varphi^{m}\left(P_{i}\right)\right)-h\right\| & =\left\|e \varphi^{m}\left(\lambda_{z}\left(P_{i}\right)\right)-h\right\| \\
& \geqslant\left\|P_{\alpha} \varphi^{m}\left(\lambda_{z}\left(P_{i}\right)\right)-P_{\alpha} h\right\| \\
& =\left\|S_{\alpha}\left(\lambda_{z}\left(P_{i}\right)-S_{\alpha}^{*} h S_{\alpha}\right) S_{\alpha}^{*}\right\| \\
& =\left\|\lambda_{z}\left(P_{i}\right)-S_{\alpha}^{*} h S_{\alpha}\right\| \\
& \geqslant\left|\omega\left(\lambda_{z}\left(P_{i}\right)-S_{\alpha}^{*} h S_{\alpha}\right)\right| \\
& =\delta .
\end{aligned}
$$

Now suppose there is an $x \neq 0$ in $\mathcal{F}_{n}$ such that $\lambda_{z}\left(\mathcal{D}_{n}\right) x=x \mathcal{D}_{n}$. We may assume that $\|x\|=1$. Since $x^{*} \lambda_{z}\left(\mathcal{D}_{n}\right)=\mathcal{D}_{n} x^{*}$ as well, we have $x^{*} x \mathcal{D}_{n}=\mathcal{D}_{n} x^{*} x$, and thus $x^{*} x \in \mathcal{D}_{n}$ by lemma 3.2 .

Take a small $\varepsilon>0$. For some $l \in \mathbb{N}$, there exists a $y_{0} \in \mathcal{F}_{n}^{l}$ such that $\left\|x-y_{0}\right\|<\varepsilon$. Then $\left\|x^{*} x-y_{0}^{*} y_{0}\right\|<\varepsilon(2+\varepsilon)$. For some $k \geqslant l$ there exists a $d \in \mathcal{D}_{n}^{k}$ such that $\left\|x^{*} x-d\right\|<\varepsilon$ and $d \geqslant 0$. Then $\left\|y_{0}^{*} y_{0}-d\right\|<\varepsilon(3+\varepsilon)$, and hence $\left\|\left|y_{0}\right|-\sqrt{d}\right\|<$ $\sqrt{\varepsilon(3+\varepsilon)}$ due to operator monotonicity of the square root function. Indeed, since $y_{0}^{*} y_{0} \leqslant d+\varepsilon(3+\varepsilon)$, we have $\left|y_{0}\right|=\sqrt{y_{0}^{*} y_{0}} \leqslant \sqrt{d+\varepsilon(3+\varepsilon)} \leqslant \sqrt{d}+\sqrt{\varepsilon(3+\varepsilon)}$ and, likewise, $\sqrt{d} \leqslant\left|y_{0}\right|+\sqrt{\varepsilon(3+\varepsilon)}$. Now, write $y_{0}=w\left|y_{0}\right|$ with $w$ a unitary in $\mathcal{F}_{n}^{l} \subseteq \mathcal{F}_{n}^{k}$. Setting $y:=w \sqrt{d}$, we have $y \in \mathcal{F}_{n}^{k}, y^{*} y=d \in \mathcal{D}_{n}^{k}$ and

$$
\|x-y\| \leqslant\left\|x-y_{0}\right\|+\left\|w\left|y_{0}\right|-w \sqrt{d}\right\|<\varepsilon+\sqrt{\varepsilon(3+\varepsilon)}=: \varepsilon^{\prime} .
$$

Let $p$ be the projection in $D_{n}^{1}$ and let $\delta>0$ be such that identity (3.3) holds. Let $g \in \mathcal{D}_{n}$ satisfy $\lambda_{z}\left(\varphi^{k}(p)\right) x=x g$. Also, let $q$ be the spectral projection of $d$ corresponding to eigenvalue $\|d\|$. Then we have

$$
\begin{aligned}
0 & =\left\|\lambda_{z}\left(\varphi^{k}(p)\right) x-x g\right\| \\
& =\left\|\left(\lambda_{z}\left(\varphi^{k}(p)\right) y-y g\right)+\left(\lambda_{z}\left(\varphi^{k}(p)\right)(x-y)-(x-y) g\right)\right\| \\
& >\left\|y\left(\lambda_{z}\left(\varphi^{k}(p)\right)-g\right)\right\|-\varepsilon^{\prime}(1+\|g\|) \\
& \geqslant \frac{1}{1+\varepsilon^{\prime}}\left\|y^{*} y\left(\lambda_{z}\left(\varphi^{k}(p)\right)-g\right)\right\|-\varepsilon^{\prime}(1+\|g\|) .
\end{aligned}
$$

We have $y^{*} y=d, d q=\|d\| q$ and $\|d\| \geqslant\left\|x^{*} x\right\|-\varepsilon=1-\varepsilon$. Thus,

$$
\begin{aligned}
\frac{1}{1+\varepsilon^{\prime}}\left\|y^{*} y\left(\lambda_{z}\left(\varphi^{k}(p)\right)-g\right)\right\|-\varepsilon^{\prime}(1+\|g\|) \\
\geqslant \frac{1-\varepsilon}{1+\varepsilon^{\prime}}\left\|q\left(\lambda_{z}\left(\varphi^{k}(p)\right)-g\right)\right\|-\varepsilon^{\prime}(1+\|g\|) \\
\geqslant \frac{1-\varepsilon}{1+\varepsilon^{\prime}} \delta-\varepsilon^{\prime}(1+\|g\|)
\end{aligned}
$$


by (3.3). Since $\varepsilon$ and $\varepsilon^{\prime}$ can be simultaneously arbitrarily small, this is a contradiction, which shows that $x=0$.

REMARK 3.5. MASAs of the form $\lambda_{z}\left(\mathcal{D}_{n}\right), z \in \mathcal{U}\left(\mathcal{F}_{n}^{1}\right)$, are called standard and are used to compute non-commutative topological entropy of certain endomorphisms $[17,26,27]$. They are abstractly characterized in [1].

For lemma 3.6, note that, given any partial isometry $S_{\alpha} S_{\beta}^{*}$ with $|\alpha|,|\beta| \geqslant 1$, there exists a $w \in \mathcal{S}_{n}$ of the form $w=S_{\alpha} S_{\beta}^{*}+\sum_{(\mu, \nu)} S_{\mu} S_{\nu}^{*}$. This is easily verified with help of the pigeonhole principle. Recall also that $E$ denotes the faithful conditional expectation from $\mathcal{O}_{n}$ onto $\mathcal{F}_{n}$.

Lemma 3.6. Let $a \in \mathcal{O}_{n}$. If $E(a v)=0$ for all $v \in \mathcal{S}_{n}$, then $a=0$.

Proof. For each projection $P_{\beta} \in \mathcal{D}_{n}, \beta \in W_{n}$, we have $0=E(a v) P_{\beta}=E\left(a\left(v P_{\beta}\right)\right)$. Thus $E\left(a S_{\alpha} S_{\beta}^{*}\right)=0$ for all $\alpha, \beta$, with $|\alpha|,|\beta| \geqslant 1$. Since the linear span of such elements $S_{\alpha} S_{\beta}^{*}$ is dense in $\mathcal{O}_{n}$ and $E$ is faithful, we conclude that $a=0$.

Theorem 3.7. If $z \in \mathcal{U}\left(\mathcal{F}_{n}^{1}\right) \backslash \mathcal{N}_{\mathcal{F}_{n}^{1}}\left(\mathcal{D}_{n}^{1}\right)$ and $a \in \mathcal{O}_{n}$ is such that $\lambda_{z}\left(\mathcal{D}_{n}\right) a=$ $a \mathcal{D}_{n}$, then $a=0$. In particular, there is no unitary $u \in \mathcal{O}_{n}$ such that $\lambda_{z}\left(\mathcal{D}_{n}\right)=$ $\operatorname{Ad}(u)\left(\mathcal{D}_{n}\right)$.

Proof. Suppose, by way of contradiction, that $\lambda_{z}\left(\mathcal{D}_{n}\right) a=a \mathcal{D}_{n}$. Then, since unitaries from $\mathcal{S}_{n}$ normalize $\mathcal{D}_{n}$, for any $v \in \mathcal{S}_{n}$ we have $\lambda_{z}\left(\mathcal{D}_{n}\right) a v=a v \mathcal{D}_{n}$. Since $\lambda_{z}\left(\mathcal{D}_{n}\right) \subseteq \mathcal{F}_{n}$, this implies that $\lambda_{z}\left(\mathcal{D}_{n}\right) E(a v)=E(a v) \mathcal{D}_{n}$. Therefore, $E(a v)=0$ by lemma 3.4 , and consequently $a=0$ by lemma 3.6.

An immediate consequence of theorem 3.7 is the existence of two MASAs of the Cuntz algebra $\mathcal{O}_{n}$ that are outer, but not inner, conjugate. In fact, theorem 3.7 implies the following stronger fact.

COROLlary 3.8. There exists an uncountable family of MASAs in $\mathcal{O}_{n}$, indexed by the cosets $\mathcal{U}\left(\mathcal{F}_{n}^{1}\right) / \mathcal{N}_{\mathcal{F}_{n}^{1}}\left(\mathcal{D}_{n}^{1}\right)$, such that each of them is outer conjugate to $\mathcal{D}_{n}$ but no two of them are inner conjugate.

To the best of our knowledge, corollary 3.8 exhibits the very first example of two MASAs in a simple purely infinite $C^{*}$-algebra that are outer, but not inner, conjugate $^{1}$.

It was shown in $[8]$ that

$$
\begin{aligned}
\left\{v \in \mathcal{U}\left(\mathcal{O}_{n}\right) \mid \lambda_{v} \in\right. & \left.\operatorname{Aut}\left(\mathcal{O}_{n}, \mathcal{D}_{n}\right)\right\} \\
& =\left\{d w \mid d \in \mathcal{U}\left(\mathcal{D}_{n}\right), w \in \mathcal{S}_{n} \text { such that } \lambda_{w} \in \operatorname{Aut}\left(O_{n}\right)\right\}
\end{aligned}
$$

On the other hand, the set $\left\{u \varphi\left(u^{*}\right) \mid u \in \mathcal{U}\left(\mathcal{O}_{n}\right)\right\}$ is dense in $\mathcal{U}\left(\mathcal{O}_{n}\right)$ by [24, theorem 3.6]. Furthermore, $\mathcal{U}_{a}\left(\mathcal{O}_{n}\right):=\left\{v \in \mathcal{U}\left(\mathcal{O}_{n}\right) \mid \lambda_{v} \in \operatorname{Aut}\left(\mathcal{O}_{n}\right)\right\}$ is a dense $G_{\delta}$-subset of $\mathcal{U}\left(\mathcal{O}_{n}\right)$ such that $\mathcal{U}\left(\mathcal{O}_{n}\right) \backslash \mathcal{U}_{a}\left(\mathcal{O}_{n}\right)$ is also dense [2, proposition 7]. In this context, we mention the following corollary.

\footnotetext{
${ }^{1}$ The authors are grateful to Mikael Rørdam for his comments on this point.
} 
COROLLARY 3.9. The following inclusion is proper:

$\left\{u d w \varphi\left(u^{*}\right) \mid u \in \mathcal{U}\left(\mathcal{O}_{n}\right), d \in \mathcal{U}\left(\mathcal{D}_{n}\right), w \in \mathcal{S}_{n}\right.$ such that $\left.\lambda_{w} \in \operatorname{Aut}\left(O_{n}\right)\right\} \subset \mathcal{U}_{a}\left(\mathcal{O}_{n}\right)$.

Proof. Suppose this is not that case. Then, for each $\alpha \in \operatorname{Aut}\left(\mathcal{O}_{n}\right)$, there exist $u \in \mathcal{U}\left(\mathcal{O}_{n}\right), d \in \mathcal{U}\left(\mathcal{D}_{n}\right)$ and $w \in \mathcal{S}_{n}$ such that $\alpha=\lambda_{u d w \varphi\left(u^{*}\right)}=\operatorname{Ad}(u) \lambda_{d w}$. Since $d w$ normalizes $\mathcal{D}_{n}$, we have $\lambda_{d w}\left(\mathcal{D}_{n}\right)=\mathcal{D}_{n}\left[13\right.$, proposition 1.4]. But then $\alpha\left(\mathcal{D}_{n}\right)=$ $\operatorname{Ad}(u)\left(\mathcal{D}_{n}\right)$, contradicting theorem 3.7.

We close this section by posing the following question. Suppose that $z \in \mathcal{U}\left(\mathcal{F}_{n}\right)$ is such that $\lambda_{z} \in \operatorname{Aut}\left(\mathcal{O}_{n}\right)$ and that there exists a $u \in \mathcal{U}\left(\mathcal{O}_{n}\right)$ such that $\lambda_{z}\left(\mathcal{D}_{n}\right)=$ $\operatorname{Ad}(u)\left(\mathcal{D}_{n}\right)$. Does this imply the existence of a $v \in \mathcal{U}\left(\mathcal{F}_{n}\right)$ such that $\lambda_{z}\left(\mathcal{D}_{n}\right)=$ $\operatorname{Ad}(v)\left(\mathcal{D}_{n}\right) ?$

\section{The outer automorphism group of $\mathcal{O}_{n}$}

In this section, we collect a few observations about the structure of the outer automorphism group of $\mathcal{O}_{n}$. We denote by $\pi: \operatorname{Aut}\left(\mathcal{O}_{n}\right) \rightarrow \operatorname{Out}\left(\mathcal{O}_{n}\right)$ the canonical surjection.

Proposition 4.1. If $d \in \mathcal{U}\left(\mathcal{D}_{n}\right)$ and $u \in \mathcal{S}_{n}$, then $\lambda_{d} \lambda_{u} \in \operatorname{Inn}\left(\mathcal{O}_{n}\right)$ if and only if there exist $v \in \mathcal{U}\left(\mathcal{D}_{n}\right)$ and $y \in \mathcal{S}_{n}$ such that $\lambda_{d}=\operatorname{Ad}(v)$ and $\lambda_{u}=\operatorname{Ad}(y)$.

Proof. If $\lambda_{d} \lambda_{u}=\operatorname{Ad}(w)$ for some $w \in \mathcal{U}\left(\mathcal{O}_{n}\right)$, then $w$ normalizes $\mathcal{D}_{n}$, and thus $w=v y$, for some $v \in \mathcal{U}\left(\mathcal{D}_{n}\right)$ and $y \in \mathcal{S}_{n}$ [23, lemma 5.4]. Hence, $\operatorname{Ad}\left(v^{*}\right) \lambda_{d}=$ $\operatorname{Ad}(y) \lambda_{u}^{-1}$ and, consequently, $\lambda_{d}=\operatorname{Ad}(v)$ and $\lambda_{u}=\operatorname{Ad}(y)$, since the intersection of $\lambda\left(\mathcal{U}\left(\mathcal{D}_{n}\right)\right)$ and $\lambda\left(\mathcal{S}_{n}\right)^{-1}$ is trivial.

The semi-direct product decomposition in proposition 4.2 is a special case of [ 20 , theorem 6.5] pertaining to a broader class of algebras. We include a short selfcontained proof, different from Matsumoto's argument.

Recall that an automorphism is aperiodic if its image in the outer automorphism group has infinite order. A Bogolubov automorphism $\lambda_{z}$ of $\mathcal{O}_{n}$ is aperiodic if and only if the corresponding unitary $z$ has infinite order. Thus, in particular, a gauge automorphism $\gamma_{t}$ is aperiodic if and only if $t$ is not a root of unity.

Proposition 4.2. The subgroup $\pi\left(\operatorname{Aut}\left(\mathcal{O}_{n}, \mathcal{D}_{n}\right)\right)$ of $\operatorname{Out}\left(\mathcal{O}_{n}\right)$ is not normal, and has the structure of a semi-direct product

$$
\pi\left(\operatorname{Aut}\left(\mathcal{O}_{n}, \mathcal{D}_{n}\right)\right)=\pi\left(\lambda\left(\mathcal{U}\left(\mathcal{D}_{n}\right)\right)\right) \rtimes \pi\left(\lambda\left(\mathcal{S}_{n}\right)^{-1}\right) .
$$

Proof. Since all aperiodic automorphisms of $\mathcal{O}_{n}$ are outer conjugate (see [21, theorem 9] and [24, theorem 3.6]), a normal subgroup of $\operatorname{Out}\left(\mathcal{O}_{n}\right)$ contains either none or all of them. Clearly, $\lambda\left(\mathcal{U}\left(\mathcal{F}_{n}^{1}\right)\right)$ contains aperiodic automorphisms $\lambda_{z}$ such that $z$ does not normalize $\mathcal{D}_{n}^{1}$. Thus, theorem 3.7 implies that $\pi\left(\operatorname{Aut}\left(\mathcal{O}_{n}, \mathcal{D}_{n}\right)\right)$ contains some, but not all, aperiodic automorphisms of $\mathcal{O}_{n}$. Consequently, it is not a normal subgroup of $\operatorname{Out}\left(\mathcal{O}_{n}\right)$.

For the semi-direct product decomposition, it suffices to note that $\pi\left(\lambda\left(\mathcal{U}\left(\mathcal{D}_{n}\right)\right)\right)$ and $\pi\left(\lambda\left(\mathcal{S}_{n}\right)^{-1}\right)$ have trivial intersection. Indeed, suppose that $d \in \mathcal{U}\left(\mathcal{D}_{n}\right), w \in \mathcal{S}_{n}$, $\lambda_{w} \in \operatorname{Aut}\left(\mathcal{O}_{n}\right)$ and $u \in \mathcal{U}\left(\mathcal{O}_{n}\right)$ are such that $\lambda_{d}=\operatorname{Ad}(u) \lambda_{w}$. Then, for each 
$p \in \mathcal{D}_{n}$, we have $p=\lambda_{d}(p)=u \lambda_{w}(p) u^{*}$, and thus $u \in \mathcal{N}_{\mathcal{O}_{n}}\left(\mathcal{D}_{n}\right)$. Hence, there exist $d_{1} \in \mathcal{U}\left(\mathcal{D}_{n}\right)$ and $w_{1} \in \mathcal{S}_{n}$ such that $u=d_{1} w_{1}\left[23\right.$, lemma 5.4]. But then $\operatorname{Ad}\left(d_{1}^{*}\right) \lambda_{d}=$ $\operatorname{Ad}\left(w_{1}\right) \lambda_{w}$, and equivalently $\lambda_{d_{1}^{*} d \varphi\left(d_{1}\right)}=\lambda_{w_{1} w \varphi\left(w_{1}^{*}\right)}$. This yields $d_{1}^{*} d \varphi\left(d_{1}\right)=1=$ $w_{1} w \varphi\left(w_{1}^{*}\right)$, and thus both $\lambda_{d}=\operatorname{Ad}\left(d_{1}\right)$ and $\lambda_{w}=\operatorname{Ad}\left(w_{1}^{*}\right)$ are inner.

We have seen in proposition 4.2 that the subgroup $\pi\left(\operatorname{Aut}\left(\mathcal{O}_{n}, \mathcal{D}_{n}\right)\right)$ is not normal in $\operatorname{Out}\left(\mathcal{O}_{n}\right)$. It follows from proposition 4.3 that the smallest normal subgroup of $\operatorname{Out}\left(\mathcal{O}_{n}\right)$ containing $\pi\left(\operatorname{Aut}\left(\mathcal{O}_{n}, \mathcal{D}_{n}\right)\right)$ is quite large.

Proposition 4.3. If $G$ is a normal subgroup of $\operatorname{Out}\left(\mathcal{O}_{n}\right)$ containing at least one aperiodic element, then

$$
\pi\left(\mathcal{Z}\left(\operatorname{Out}\left(\mathcal{O}_{n}\right)\right) \cup \lambda\left(\mathcal{U}\left(\mathcal{F}_{n}\right)\right)^{-1} \cup\left\{\alpha \in \operatorname{Aut}\left(\mathcal{O}_{n}\right) \mid \alpha \text { aperiodic }\right\}\right) \subseteq G .
$$

In particular, (4.1) holds with $G$, the commutator subgroup of $\operatorname{Out}\left(\mathcal{O}_{n}\right)$.

Proof. Since all aperiodic automorphisms of $\mathcal{O}_{n}$ are outer conjugate to one another (see [21, theorem 9] and [24, theorem 3.6]), group $G$ contains classes of all of them. Now, let $\alpha \in \operatorname{Aut}\left(\mathcal{O}_{n}\right)$ and suppose that there exists an aperiodic $\beta \in \operatorname{Aut}\left(\mathcal{O}_{n}\right)$ such that $\alpha \beta$ is aperiodic. Then there exists a $\psi \in \operatorname{Aut}\left(\mathcal{O}_{n}\right)$ such that $\pi(\alpha \beta)=$ $\pi\left(\psi \beta \psi^{-1}\right)$, and consequently $\pi(\alpha)=\pi\left(\alpha \beta \beta^{-1}\right)=\pi\left(\psi \beta \psi^{-1}\right) \pi\left(\beta^{-1}\right)$ belongs to $G$. Now, let $\alpha \in \operatorname{Aut}\left(\mathcal{O}_{n}\right)$ be such that either $\pi(\alpha) \in \mathcal{Z}\left(\operatorname{Out}\left(\mathcal{O}_{n}\right)\right)$ or $\alpha \in \lambda\left(\mathcal{U}\left(\mathcal{F}_{n}\right)\right)^{-1}$. Take an aperiodic gauge automorphism $\gamma_{t}$ such that $\pi\left(\gamma_{t}^{m}\right) \neq \pi\left(\alpha^{-m}\right)$ for all $m \in \mathbb{N}$. Since $\pi(\alpha)$ commutes with $\pi\left(\gamma_{t}\right)$, the product $\alpha \gamma_{t}$ is again aperiodic. This shows the first claim of the proposition. For the remaining one, simply note that if $\theta \in \operatorname{Aut}\left(\mathcal{O}_{n}\right)$ is aperiodic, then $\theta^{2}$ is outer conjugate to $\theta$. Thus, $\pi\left(\theta^{2}\right)=\pi\left(\psi \theta \psi^{-1}\right)$ for some $\psi \in \operatorname{Aut}\left(\mathcal{O}_{n}\right)$, and hence $\pi(\theta)=\pi\left(\theta^{2}\right) \pi\left(\theta^{-1}\right)=\pi\left(\psi \theta \psi^{-1} \theta^{-1}\right)$ belongs to the commutator subgroup $\left[\operatorname{Out}\left(\mathcal{O}_{n}\right), \operatorname{Out}\left(\mathcal{O}_{n}\right)\right]$.

REMARK 4.4. No class of non-trivial gauge automorphism of $\mathcal{O}_{n}$ belongs to the centre of $\operatorname{Out}\left(\mathcal{O}_{n}\right)$. Indeed, consider the unitary $u=S_{11} S_{121}^{*}+S_{121} S_{11}^{*}+P_{122}+P_{2}$ in $\mathcal{S}_{2}$, discussed in [11, theorem 5.2]. Then $\lambda_{u}$ is an automorphism of $\mathcal{O}_{2}$ such that $\lambda_{u}^{2}=$ id. For $1 \neq t \in U(1)$ we have $\lambda_{u} \gamma_{t} \lambda_{u}^{-1} \gamma_{t}^{-1}=\lambda_{w}$, with $w=t \lambda_{u}\left(P_{121}\right)+$ $\bar{t} \lambda_{u}\left(P_{11}\right)+P_{122}+P_{2}$. The automorphism $\lambda_{w}$ of $\mathcal{O}_{2}$ is outer, for otherwise there exists a $d \in \mathcal{U}\left(\mathcal{D}_{2}\right)$ such that $w=d \varphi\left(d^{*}\right)$. But then $w$, viewed as a function on $X_{2}$, would take value 1 at the infinite word $111 \ldots$ (fixed by the shift on $X_{2}$ ). However, this is not the case. A similar argument applies to all $n$, with a suitably modified $u \in \mathcal{S}_{n}$ (cf. [11, theorem 5.2]).

This is in stark contrast with what happens for the weak closure $M$ of $\mathcal{O}_{n}$ in the Gelfand-Naimark-Segal representation of the canonical Kubo-Martin-Schwinger state $\omega=\tau \circ E$, which is the approximately finite-dimensional factor of type $I I I_{1 / n}$. Indeed, gauge automorphisms of $\mathcal{O}_{n}$ extend to $M=\pi_{\omega}\left(\mathcal{O}_{n}\right)^{\prime \prime}$ [7], thereby providing the $(2 \pi / \log (n)$-periodic) modular automorphisms (with respect to the normal extension of $\omega$ ), which then lie in the centre of $\operatorname{Out}(M)$ by the Connes-RadonNikodým theorem [3, theorem 1.2.8]. It also follows from this argument that the automorphism $\lambda_{u}$ above does not extend to $M$ (i.e. it is not normal).

REMARK 4.5. Those classes under inner equivalence of all automorphisms of $\mathcal{O}_{2}$ known to us at the moment belong to the commutator subgroup of $\operatorname{Out}\left(\mathcal{O}_{2}\right)$. For example, consider the unitary $u \in \mathcal{S}_{2}$ discussed in remark 4.4. Then, for an aperiodic 
gauge automorphism $\gamma_{t}$, the automorphism $\lambda_{u} \gamma_{t}$ is aperiodic as well. Indeed, since $\lambda_{u}$ has order 2 , it suffices to show that all even powers of $\lambda_{u} \gamma_{t}$ are outer. But we have $\lambda_{u} \gamma_{t} \lambda_{u} \gamma_{t}=\lambda_{v}$, with $v=t^{3} \lambda_{u}\left(P_{121}\right)+t \lambda_{u}\left(P_{11}\right)+P_{122}+P_{2}$, and $\lambda_{v}$ is aperiodic for the same reason as given in remark 4.4. Now, the same argument as in proposition 4.3 gives the conclusion that $\lambda_{u}$ is a commutator modulo an inner automorphism of $\mathrm{O}_{2}$.

We close this paper with a few simple, albeit potentially useful, observations about the outer automorphism group of $\mathcal{O}_{2}$. First of all, it is worth noting that by combining some of the results from [21] and [8] one easily obtains the following.

Proposition 4.6. Every element of infinite order in $\operatorname{Out}\left(\mathcal{O}_{2}\right)$ is a product of two involutions in the commutator subgroup. In particular, both $\left[\operatorname{Out}\left(\mathcal{O}_{2}\right), \operatorname{Out}\left(\mathcal{O}_{2}\right)\right]$ and $\operatorname{Out}\left(\mathrm{O}_{2}\right)$ are generated by elements of finite order.

Proof. We consider the inner equivalence classes of the automorphisms $\lambda_{A}$ and $\lambda_{F}$ defined in $[8, \oint 5.3]$. As shown therein, the commutator $\lambda_{F} \lambda_{A} \lambda_{F} \lambda_{A}^{-1}$ has infinite order in Out $\left(\mathcal{O}_{2}\right)$ and is a product of two involutions. The conclusion follows immediately, as every aperiodic automorphism is a conjugate of such commutator.

We believe that the same result holds true for $\operatorname{Out}\left(\mathcal{O}_{n}\right)$ for all $n \geqslant 2$, with a suitable modification of the lengthy computations in [8]. As this falls outside the scope of the present work, we leave the task to the interested reader.

COROLlary 4.7. The normal subgroup of $\operatorname{Out}\left(\mathcal{O}_{2}\right)$ generated by $\lambda\left(\mathcal{S}_{2}\right)^{-1}$ is generated by elements of finite order.

Proof. There are two possible cases.

(1) If $\pi\left(\lambda\left(\mathcal{S}_{2}\right)^{-1}\right)$ is not contained in the commutator subgroup of $\operatorname{Out}\left(\mathcal{O}_{2}\right)$, then there is an element in $\pi\left(\lambda\left(\mathcal{S}_{2}\right)^{-1}\right)$ but not in the commutator subgroup, say $g$, necessarily of finite order. Moreover, $g h$ must have finite order for any $h$ of infinite order in $\pi\left(\lambda\left(\mathcal{S}_{2}\right)^{-1}\right)$. Accordingly, any $h$ of infinite order in $\pi\left(\lambda\left(\mathcal{S}_{2}\right)^{-1}\right)$ can be written as $g^{-1}(g h)$, a product of finite order elements.

(2) On the other hand, if $\pi\left(\lambda\left(\mathcal{S}_{2}\right)^{-1}\right)$ is in $\left[\operatorname{Out}\left(\mathcal{O}_{2}\right), \operatorname{Out}\left(\mathcal{O}_{2}\right)\right]$, then any element of infinite order in $\pi\left(\lambda\left(\mathcal{S}_{2}\right)^{-1}\right)$ is a product of two conjugates of involutions in $\pi\left(\lambda\left(\mathcal{P}_{2}\right)^{-1}\right)$, by proposition 4.6 .

To the best of our knowledge, the following result provides the first non-trivial structural result about the rather mysterious group $\lambda\left(\mathcal{S}_{2}\right)^{-1}$. Recall that a group $G$ is called almost simple if there exists a non-abelian simple group $H$ such that $H \subseteq G \subseteq \operatorname{Aut}(H)$.

Proposition 4.8. The group $\left.\lambda\left(\mathcal{S}_{2}\right)^{-1}\right|_{\mathcal{S}_{2}}$ is almost simple.

Proof. Clearly, any automorphism of the form $\lambda_{w}$, with $w \in \mathcal{S}_{2}$, restricts to an automorphism of $\mathcal{S}_{2}$. Also, $\mathcal{S}_{2}$, being isomorphic to the Thompson group $V$, is simple [22, proposition 9.6] and, in particular, its centre is trivial. Thus, one has inclusions

$$
\left.\mathcal{S}_{2} \simeq \operatorname{Inn}\left(\mathcal{S}_{2}\right) \subseteq \lambda\left(\mathcal{S}_{2}\right)^{-1}\right|_{\mathcal{S}_{2}} \subseteq \operatorname{Aut}\left(\mathcal{S}_{2}\right),
$$

and the conclusion follows. 
Of course, one might wonder whether the kernel of the restriction map $\lambda\left(\mathcal{S}_{2}\right)^{-1} \rightarrow$ $\left.\lambda\left(\mathcal{S}_{2}\right)^{-1}\right|_{\mathcal{S}_{2}}$ is trivial. The analogous statement for $\mathcal{P}_{n}$ has been discussed in [9] (see the paragraph following proposition 3.6 therein).

\section{Acknowledgements}

R.C., J.H.H. and W.S. were supported through the programme 'Research in Pairs' by the Mathematisches Forschungsinstitut Oberwolfach in 2013. J.H.H. was supported by the Basic Science Research Program through the National Research Foundation of Korea funded by the Ministry of Education, Science and Technology (Grant no. 2012R1A1A2039991). W.S. was partly supported by the Villum Fonden Research Network 'Experimental mathematics in number theory, operator algebras and topology' (2012-2015), and the FNU project grant 'Operator algebras, dynamical systems and quantum information theory' (2013-2015).

\section{References}

1 E. J. Beggs and P. Goldstein. Maximal abelian subalgebras of $\mathcal{O}_{n}$. C. R. Acad. Sci. Paris Sér. I 24 (2002), 26-32.

2 O. Bratteli and A. Kishimoto. Homogeneity of the pure state space of the Cuntz algebra. J. Funct. Analysis 171 (2000), 331-345.

3 A. Connes. Une classification des facteurs de type III. Annales Scient. Éc. Norm. Sup. 6 (1973), 133-252.

4 A. Connes. Outer conjugacy classes of automorphisms of factors. Annales Scient. Éc. Norm. Sup. 8 (1975), 383-419.

5 A. Connes. Periodic automorphisms of the hyperfinite factor of type $\mathrm{II}_{1}$. Acta Sci. Math. (Szeged) 39 (1977), 39-66.

6 R. Conti. Automorphisms of the UHF algebra that do not extend to the Cuntz algebra. $J$. Austral. Math. Soc. 89 (2010), 309-315.

7 R. Conti and C. Pinzari. Remarks on the index of endomorphisms of Cuntz algebras. J. Funct. Analysis 142 (1996), 369-405.

8 R. Conti and W. Szymański. Labeled trees and localized automorphisms of the Cuntz algebras. Trans. Am. Math. Soc. 363 (2011), 5847-5870.

9 R. Conti and W. Szymański. Automorphisms of the Cuntz algebras. In Progress in operator algebras, noncommutative geometry, and their applications: proceedings 4 th annual meeting of the European noncommutative geometry network, Bucharest, 2011, pp. 1-15 (Bucharest: Theta, 2012).

10 R. Conti, J. H. Hong and W. Szymański. The restricted Weyl group of the Cuntz algebra and shift endomorphisms. J. Reine Angew. Math. 667 (2012), 177-191.

11 R. Conti, J. H. Hong and W. Szymański. The Weyl group of the Cuntz algebra. Adv. Math. 231 (2012), 3147-3161.

12 J. Cuntz. Simple $C^{*}$-algebras generated by isometries. Commun. Math. Phys. 57 (1977), $173-185$.

13 J. Cuntz. Automorphisms of certain simple $C^{*}$-algebras. In Quantum fields: algebras, processes (ed. L. Streit), pp. 187-196 (Springer, 1980).

14 J. Cuntz. K-theory for certain $C^{*}$-algebras. Annals Math. (2) 113 (1981), 181-197.

15 M. Enomoto, H. Takehana and Y. Watatani. Automorphisms on Cuntz algebras. Math. Japon. 24 (1979), 231-234.

16 J. Glimm. On a certain class of operator algebras. Trans. Am. Math. Soc. 95 (1960), $318-340$.

17 J. H. Hong, A. Skalski and W. Szymański. On invariant MASAs for endomorphisms of the Cuntz algebras. Indiana Univ. Math. J. 59 (2010), 1873-1892.

18 M. Izumi. Finite group actions on $C^{*}$-algebras with the Rohlin property. I. Duke Math. J. 122 (2004), 233-280. 
19 A. Kishimoto. The Rohlin property for shifts on UHF algebras and automorphisms of Cuntz algebras. J. Funct. Analysis 140 (1996), 100-123.

20 K. Matsumoto. Orbit equivalence of topological Markov shifts and Cuntz-Krieger algebras. Pac. J. Math. 246 (2010), 199-225.

21 H. Nakamura. Aperiodic automorphisms of nuclear purely infinite simple $C^{*}$-algebras. Ergod. Theory Dynam. Syst. 20 (2000), 1749-1765.

22 V. Nekrashevych. Cuntz-Pimsner algebras of group actions. J. Operat. Theory 52 (2004), 223-249.

23 S. C. Power. Homology for operator algebras. III. Partial isometry homotopy and triangular algebras. New York J. Math. 4 (1998), 35-56.

24 M. Rørdam. Classification of inductive limits of Cuntz algebras. J. Reine Angew. Math. 440 (1993), 175-200.

25 M. Sabok. Completeness of the isomorphism problem for separable $C^{*}$-algebras. Preprint, 2013. (Available at http://arxiv.org/abs/1306.1049.)

26 A. Skalski. Noncommutative topological entropy of endomorphisms of Cuntz algebras. II. Publ. RIMS Kyoto 47 (2011), 887-896.

27 A. Skalski and J. Zacharias. Noncommutative topological entropy of endomorphisms of Cuntz algebras. Lett. Math. Phys. 86 (2008), 115-134.

(Issued 3 April 2015) 